\title{
Optimal Queueing Policies for Fast Packet Switching of Mixed Traffic
}

\author{
Geert A. Awater and Frits C. Schoute
}

\begin{abstract}
The power of ATM (asynchronous transfer mode) is its ability to provide bandwidth on demand: different sources can have different bandwidth requirements. Sources also differ in performance requirements: some ask for minimal delay variations whereas others must have extremely low cell loss probabilities. This paper shows how these complementary performance requirements can be exploited with an LDOLL (low delay or low loss) queue, where sources get either service priority or storage priority. The space of possible LDOLL queueing policies is very large: even after a justified reduction, the size is still of order $O\left(2^{Q^{2}}\right), Q$ being the maximum number of ATM cells in the LDOLL queue. Using Markov decision theory and concepts of linear programming, we arrive at only $Q$ so-called efficient solutions. These are the LDOLL threshold policies, which are conceptually appealing, robust in performance, and practical from the implementation viewpoint.
\end{abstract}

\section{INTRODUCTION}

$\mathrm{F}$ AST packet switching techniques clearly provide the flexibility for integration of mixed traffic streams, such as voice data, and video. Due to a provision for the stochastic bandwidth requirements of some traffic sources, it is not so clear whether a reasonable degree of utilization of switching and transmission resources can be achieved. That is, the primary benefit of fast packet switching lies in its flexibility to serve different traffic streams [3]. However, as a second benefit, we want to design here a method that also uses capacity efficiently.

We concentrate on ATM (asynchronous transfer mode), the emerging CCITT standard for fast packet switching, and analyze how much capacity improvement can be realized by exploiting the fact that some sources require low delay variation whereas other sources require low loss probabilities for ATM cells. In the CCITT SG XVIII ISDN experts meeting in Geneva, January 1990, it was decided that the ATM cell header should have a cell loss priority (CLP) bit [1]. The CLP bit creates a possibility to distinguish between two types of cells.

The performance of the ATM network, as exposed to the user, depends primarily on the call acceptance probability. Methods of resource allocation usually decide to accept an ATM connection if the resulting cell loss probability of the aggregate input traffic remains below a preset maximum value. The expected cell delay is considered of minor importance, since it will be very small with the proposed ATM-transmission speeds [2].

If a higher cell loss probability can be tolerated, this should be specified in the cell header, e.g., by using the CLP bit. Hereby, two classes of traffic are introduced. The first class encompasses traffic requiring low loss probability. Traffic of the

Manuscript received April 25, 1990; revised November 12, 1990 The authors are with the Department of Electrical Engineering. TVS Group, Delft University of Technology, 2600 GA Delft. Netherlands. IEEE Log Number 9042048. other class gives in on loss probability but gets a lower delay variance in return.

Sources that generate traffic, whose time relation needs to be restored after passage through the asynchronous transfer network, benefit from a low delay variance. This is so since delay dejitter buffers in the terminal equipment can be kept smaller if the transnetwork delay variance is small. Traffic sources that generate traffic for which the integrity is of prime importance, on the other hand, will prefer a low probability of cell loss. An example of the latter is (machine-oriented) data traffic, an example of the first is (human-oriented) audio/visual traffic.

With integrated circuit technology, one can realize buffers for several hundreds of ATM cells on one chip. These buffers, together with on-chip control logic, can be used to implement LDOLL (low delay or low loss) queues. An LDOLL queueing policy favors low delay cells in service priority and low loss cells in storage priority. The main contributions of this paper are: computation of optimal LDOLL queueing policies and evaluation of the benefits of the LDOLL queue in the context of mixed traffic.

To describe the sources of different traffic streams, it should be realized that a cell transmission takes a few microseconds, a burst of cells (activity period of a source) lasts, say, a fraction of a second, and the connection between source and destination can stand for several minutes. Therefore, at least two authors [13], [7] have introduced a three-level model for modeling mixed traffic streams. Following [13], we call the three levels, respectively, TRANSMISSION, ACTION, and CONNECTION. In this reference, as well as in [14], a pictorial presentation of the three-level model can be found. Hui [7] uses the terms CELL, BURST, and CALL level. Filipiak [5] calls these levels "layers" and structures the analysis further by also defining network levels.

Connections do not always need the full bandwidth for the whole duration of the connection. Moreover, the bandwidth requirement usually differs for the go or return path of a connection. The bursty character of some traffic sources or the variable bit rate (VBR) of some video coders is best described at the intermediate time scale of the ACTION level. For example, in the case of a file transfer, a burst of packets is transmitted. Termination of the file transfer does not necessarily imply immediate termination of the connection because more data may be exchanged subsequently. This implies an on/off pattern at the ACTION level. In the case of a VBR coder, the cell rate (i.e., bandwidth) may change every $1 / 25 \mathrm{~s}$ (resp. $1 / 30 \mathrm{~s}$ ), once every video frame.

The emphasis of this paper will be on the TRANSMISSION level, where the LDOLL queue operates. Loading at the TRANSMISSION level is determined by the sum of the activities of the admitted connections. With its buffers, the LDOLL queue can allow for short periods of overload. This could pro- 
vide an alternative for burst blocking at the ACTION level, as modeled in [7]. The relative frequency and duration of overloads, thereby the fractions of cells that are not delivered properly (cell loss due to buffer overflow or excessive cell delay variation), can be controlled by acceptance rules at the CONNECTION level.

Hence, different methods of traffic control can be discerned per level. At the TRANSMISSION level, by an LDOLL queueing policy; at the ACTION level, possibly by flow control (not discussed in this paper); and the CONNECTION level, by call acceptance control. All methods participate to realize a high utilization of resources but, at the same time, an acceptably small probability of cell loss or excessive delay variations. At the bottom level, we wish to contribute with the optimization of LDOLL queueing policies to strategies for improving the quality of service for mixed traffic.

A general model of an ATM switching element is presented in Section II. A Markov model of the LDOLL queue (which can be implemented in the switching element) is created in Section III. Section IV deals with finding optimal LDOLL queueing policies for different mixes of traffic streams. In Section V, the value of LDOLL policies follows from comparison with FIFO (first in first out) and the benefits of mixed traffic follow from comparison with the extremes of only low delay traffic (e.g., video conferencing) and only low loss traffic (e.g., file transfers). The conclusions are in Section VI.

\section{ATM Switching Element Model}

An ATM switching element might be considered the elementary building block of an ATM network. The element has $N$ ATM input links and $M$ ATM output links. In our model, all links of the switching element operate synchronously; the in terval between two successive (idle) cell arrivals we call a time slot. A cell arriving via an input link can be transferred to any of the $M$ output links. In the switching element, buffering may be employed to reduce cell loss during periods of high cell traffic intensity. As an example, consider a packet switch architecture with Banyan topology (see, e.g., [15]) that can be built using a number of ATM switching elements with $N=2$ and $M=2$. If the element contains a set of cell buffers, a buffered Banyan network is obtained. Fig. 1 is a graphical representation of the switching element.

The cell receivers ( $C R_{1}$ through $C R_{N}$ ) deserialize an incoming cell and store it after reception into input buffers $I B_{1} \ldots N$. Deserialization makes it possible to examine the header information, and to process ATM cells in parallel. This allows practical switching elements to cope with the high switching speeds (millions of cells per second). The functional entity that is designated with CE (cell enqueuer) transfers the nonempty cells from the input buffers to the cell buffers (CB), according to some enqueueing policy. The cell server (CS) takes cells out of the buffers obeying a service policy, and stores them in the output buffers $O B_{1} \cdots O B_{N}$. The output buffers transfer cells to the cell transmitters, $C T_{1} \cdots C T_{N}$, that serialize it and put it onto their corresponding outgoing link. Every time slot, cell service takes place prior to the enqueueing of a new cell into the cell buffers. The CS optionally bypasses the cell enqueuer and the buffers. This situation occurs, for example, if the CS finds all cell buffers empty and the input buffers hold at least one cell. Also, a switching element without buffering can be modeled with this option.

The switching function of the element may be performed by

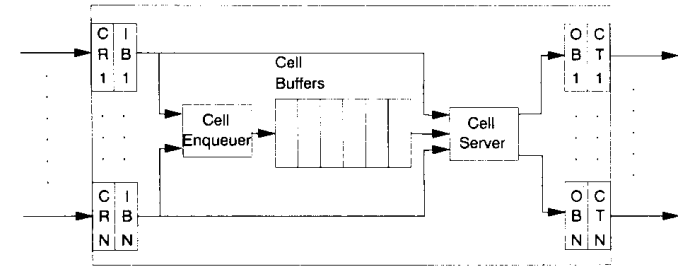

Fig. 1. Functional schematic of an ATM switching element.

either the cell enqueuer or by the cell server. If we use the general switching element to model a space division switch with output buffers (as in [8]), a partition of the cell buffer is reserved for cells with identical destination links. The CE routes an incoming cell to the appropriate buffer partition. In the input buffer model of the same authors, on the other hand, a buffer partition is allocated for every input link. In this case, the switching function is attained by the CS, which retrieves cells from all buffer partitions and relays them to their destination output links.

Note that, even if the cells were transferred from the input to the output buffers infinitely fast, the (de)serialization of cells introduces a delay of one time slot, i.e., the time needed for transmission of an entire cell. However, if we assume that enqueueing and subsequent serving takes a single time slot or more, the minimal delay introduced by the switching element is two time slots.

\section{LDOLL Queueing Model}

In this paper, we shall focus our attention to switching elements that have a separate logical buffer partition for every output link. One buffer for one output link can be modeled as a switching element that has $M=1$. The obtained results are also of significance for switching elements that have output links with shared buffers. We will show how, assuming certain conditions, the LDOLL queue population can be modeled as a finite discrete-time Markov chain. The unit of time of this chain will be one time slot. The chain starts operation at time $t=0$.

The queue holds cells requiring low delay variance, and cells that must not have a high loss probability. In the next section, we will see that cells that have a low average delay also have a low delay variance. Because delay is more tractable in our formulation of the problem, we will concentrate on the expected delay instead of delay variance. The type of cells requiring low delay (variance) will be referred to as $L D$, for the other kind this is $L L$.

Before we devise a Markov model of the LDOLL queue, the following subjects must be described:

- The cell arrival process,

- The enqueueing policy,

- The service policy.

At an input buffer $i, i=1,2, \cdots, N$, at the beginning of every time slot, one out of three possible events occurs; either a $L D$ cell, a $L L$ cell, or an empty cell pops up. We assume that the individual traffic streams at the CONNECTION level are mixed so profoundly at the TRANSMISSION level that these events do not depend on the cell arrival process history. Bursts of arrivals take place in the slower time scale of the ACTION level. Thus, the arrival statistics of a cell of any type at the TRANSMISSION level can be modeled as a Bernoulli process: $\operatorname{Pr}\{L D$ or $L L$ cell arrival $\}=p$, where $p \in[0,1]$ denotes the traffic intensity on the inlet link of the input buffer. We assume 
that all input links carry the same traffic intensity, i.e., $p$ is independent of $i$.

In general, cell arrival probabilities differ per input link and change in time. In that case, one should write $p_{i}(t)$ instead of $p$. The mixing ratio, which is defined in the next paragraph, could be dependent in the same way, i.e., $r_{i}(t)$ instead of $r$. However, for clarity of exposition of the LDOLL queue, we consider $p$ and $r$ as constants. The more general case is analyzed in [12]. In this reference, one finds a combination of simulation at the ACTION level and computation at the TRANSMISSION level.

We state that the probability that an arriving cell is of type $L D$, provided that it is a nonempty cell, equals $r, r \in[0,1]$. Again we assume that $r$ is independent of the history of the arrival process, and identical for all input links. Let $A_{L D}(t)$ and $A_{L L}(t)$ be the stochastic variables that denote the number of $L D$ cells and the number of $L L$ cells, respectively, that are present in the input buffers at the beginning of time slot $t$. We will omit the time dependency when it is implicitly clear that the current time slot is meant. $A_{L D}$ and $A_{L L}$ have a discrete, trinomial joint probability distribution.

$$
\begin{aligned}
\operatorname{Pr}\left\{A_{L D}=i, A_{L L}=j\right\} \\
=\frac{N !}{(N-i-j) ! i ! j !} \\
\quad \cdot(r p)^{i} \cdot((1-r) p)^{j} \cdot(1-p)^{N-i-j} .
\end{aligned}
$$

The state of the LDOLL queue is defined by the tuple $\left[n_{L D}(t)\right.$, $\left.n_{L L}(t)\right]$, the number of $L D$ cells and $L L$ cells in the queue at the beginning of time slot $t$. These integer numbers satisfy the constraints: $0 \leq n_{L D}(t)+n_{L I}(t) \leq Q$, where $Q$ is the (fixed) size of the queue. Again, the time dependency will be dropped when no ambiguities can arise. For reasons of brevity, all states can be identified by a unique number $X \in\{1 \cdots \Xi\}$ using a bijective mapping function $\varphi$. Here $\Xi=\frac{1}{2} \cdot(Q+1) \cdot(Q+$ 2 ) is the number of states.

$$
X(t)=\varphi\left(n_{L D D}(t), n_{I L . L}(t)\right) .
$$

If we let $S_{L D}(t)$ and $S_{L L}(t)$ denote the number of $L D$ and $L L$ cells served during time slot $t$, the enqueueing policy is described by the following rules:

$$
\begin{aligned}
n_{L L}(t+1)= & \operatorname{Max}\left\{Q, n_{L L}(t)-S_{L L}(t)+A_{L L}(t)\right\} \\
n_{L D}(t+1)= & \operatorname{Max}\left\{Q-n_{L L}(t+1), n_{L D}(t)\right. \\
& \left.-S_{L D}(t)+A_{L D}(t)\right\} .
\end{aligned}
$$

Verbally, $L L$ cells get storage priority over $L D$ cells. New $L L$ cells are allowed to replace stored $L D$ cells, but they cannot supersede other $L L$ cells. The remaining empty space in the queue is allocated to $L D$ cells. Since the enqueueing policy rules only describe the dynamics of the number of cells, they do not dictate which specific $L D$ cells must be replaced. If we define the delay of a replaced $L D$ cell to be 0 , one can easily see (Appendix B) that the oldest $L D$ cells (those with the longest sojourn time in the queue) must be replaced first in order to minimize the delay for $L D$ cells that are eventually served. This enqueueing policy clearly minimizes the loss probability of $L L$ cells as well as the delay of $L D$ cells.

In the case of the service policy, it is not so obvious which scheme should be used, except that per cell type service should be in a first come, first served (FCFS) fashion. Therefore, in this section we will give a specification of the service policy that is as general as possible. In fact, it may be nondeterministic, nonstationary (i.e., time-dependent) and non-Markovian (i.e., dependent on the history of the system). In a specific state $x$, at the beginning of time slot $t$, the cell transmitter may take an action $A(t)$ out of the state-dependent time-invariant set of possible actions $K(x)$. Here, $A(t)$ is a short denotation for the service tuple $\left[S_{L D}(t), S_{L L}(t)\right]$. The action sets for $M=1$ are:

$$
K(x)= \begin{cases}\{(0,0)\} & \text { if } n_{L D}=0 \text { and } n_{L L}=0 \\ \{(1,0)\} & \text { if } n_{L D}>1 \text { and } n_{L L}=0 \\ \{(0,1)\} & \text { if } n_{L D}>1 \text { and } n_{L L}=1 \\ \{(1,0),(0,1)\} & \text { otherwise. }\end{cases}
$$

Any specific service policy $R$ is characterized by the set that contains the probabilities of all actions that are possible in a state, for every time slot, for every state, and for every feasible system history. More precisely, it is a set of functions $\beta$ that are defined as

$$
\beta(x, a, h, t)=\operatorname{Pr}\{A(t)=a \mid X(t)=x, \quad H(t-1)=h\}
$$

with

$$
\left\{\begin{array}{l}
a \in K(x) \\
H(t-1)=\{X(t-1), A(t-1)\} \cup H(t-2) \\
H(0)=\varnothing \\
t=0,1, \cdots
\end{array}\right.
$$

and

$$
\beta \text { satisfying: } \sum_{a \in K(x)} \beta(x, a, h, t)=1 .
$$

With these definitions, we are now able to construct the matrix of state transition probabilities at time $t$, given the history up to $t$, under policy $R: P(R, h, t)$. To that end, we consider the system being in intermediate state $z$, just after cell service has taken place. Then, due to the arrival process and independently of the service policy, the system will be driven to state $y$ with probability $T_{z y}$, where

$$
T_{\exists y}=\sum_{i, j \in S_{: v}} \operatorname{Pr}\left\{A_{L D}=i, A_{L L}=j\right\}
$$

where

$$
\begin{aligned}
S_{z y} & =\{i, j \mid 0 \leq i, j \leq N, u=\operatorname{Max}(Q-v, m+i), \\
v & =\operatorname{Max}(Q, n+j)\}
\end{aligned}
$$

with

$$
\varphi(m, n)=z \text { and } \varphi(u, v)=y .
$$

That is, $S_{z y}$ is the set of all arrivals that transfer the system from intermediate state $z$ to state $y$. The service policy is incorporated in the transition matrix since the intermediate state $z$ depends both on the departure state $X(t)$, say $x$ and the action $A(t)$ taken by the cell server in that state, say $a$. So we write $z(x, a)$ and use it in order to obtain the transition matrix elements $P_{x y}(R$, $h, t)$, that represent the one-step transition probabilities from 
state $x$ to state $y$

$$
P_{x y}(R, h, t)=\sum_{a \in K(x)} T_{z(x, a), y} \cdot \beta(x, a, h, t)
$$

with

$$
z(x, a)=\varphi((m, n)-a) \quad \text { if } x=\varphi(m, n) .
$$

Note that if $\beta$

- is independent of $h, X(t)$ is a Markov chain.

- is independent of $h$ and $t, X(t)$ is a stationary Markov chain.

- is independent of $h$ and $t$, and if $\forall x: \exists a^{*}: \beta\left(x, a^{*}\right)=1$, we have a deterministic policy.

Note that, in all three cases, the Markov chain is irreducible and finite, provided that the system does not degenerate by letting $p$ or $r$ reach their ultimate values of 0 or 1 . The irreducibility follows from the fact that all states communicate. This is because all states are reachable, starting with an empty queue. Also, from any state, there is a nonzero probability of returning to the empty queue state again. The system is finite because the queue length is bounded.

In the latter two cases, we can determine the equilibrium probabilities of the queue under policy $R, \pi_{x}(R) \triangleq \lim _{t \rightarrow \infty}$ $\operatorname{Pr}_{R}\{X(t)=x\}$ by solving:

$$
\left\{\begin{array}{l}
\sum_{x} \pi_{x}(R) \cdot P_{x y}(R)=\pi_{y}(R) \\
\sum_{x} \pi_{x}(R)=1 .
\end{array}\right.
$$

In this paper, the delay and loss characteristics of the LDOLL queue, for both types of cells, are improved by incorporating the cell buffer bypass mentioned in Section III. If all cell buffers are empty after cell service has taken place and there is a cell in one of the buffers, that cell is transferred directly to the output buffer. If there is more than one cell in the $I B$ 's, $L D$ cells acquire precedence over $L L$ cells. The bypass mechanism introduces a dependency of the arrival probabilities on the state yielding different $P_{0 y}$ in (3.7).

\section{Optimal Service Policy}

Markov decision theory studies the control of a Markov chain, using a control policy as defined in the preceding section. Procedures have been found that optimize object functions of different kinds. The object function that we are interested in here is referred to as the expected average cost criterion. This function is defined for every policy and starting state $x_{0}(\operatorname{Pr}\{X(0)$ $\left.=x_{0}\right\}=1$ ) as

$$
\begin{aligned}
\Psi\left(R, x_{0}\right) \triangleq & \lim _{T \rightarrow \infty} \frac{1}{T+1} \cdot \sum_{t=0}^{T} \sum_{x} \pi_{x}(t) \\
& \cdot \sum_{a \in K(x)} w_{x a} \cdot \beta(x, a, h, t) .
\end{aligned}
$$

Here, $\pi_{x}(t) \triangleq \operatorname{Pr}_{R}\{X(t)=x\}$ and $w_{x a}$ is the cost that is incurred if action $a$ (as prescribed by policy $R$ ) is taken, given that the system is in state $X(t)=x$. This amount is assumed to be independent of time. When minimizing $\Psi$, we do not have to take all policies into account. In Markov decision theory, it is proven that the policy that optimizes $\Psi$ belongs to the class of deterministic policies (see, e.g., [4], p. 25). Thus, we only need to restrict the range of $\beta$ to the values 0 and 1 , and let $\beta$ depend on $a$ and $x$ only. For now, we suppose that we are able to construct an objective function for the minimization of both the $L D$ cell delay variance and the $L L$ cell loss probability, expressed in the form of (4.1). If not, we are likely to obtain nondeterministic or time- or history-dependent policies, as studied in [11] for instance. The disadvantage of such policies is that they are implemented with more overhead. Therefore, we are willing to optimize an approximation that takes the form of (4.1) if the objective function itself does not.

Because the LDOLL Markov chain is ergodic and irreducible under every conceivable deterministic policy, (4.1) reduces to (4.2), irrespective of starting state $x_{0}$

$$
\Psi(R)=\sum_{x} \pi_{x}(R) \cdot w_{x}(R)
$$

where $w_{x}(R)$ is a short notation for the cost incurred in state $x$ under policy $R$. Apart from the question of the applicability of this form, we also face the problem of the dimensionality of the optimization problem, which is still too large [of order $O\left(2^{Q^{2}}\right)$ ] to allow us to find the optimum by means of an exhaustive search.

In Markov decision theory, the second problem has been resolved in several ways. Manne (in [10]) showed that the optimal policy derivation for our case can be formulated as a linear programming (LP) problem. The advantages of the LP optimization approach, with respect to other methods (see, e.g., [4], ch. 6), are:

A) Often LP routines are available, which usually exploit the sparsity in the coefficients of the constraint equations. We had the opportunity to use a dedicated LP machine, with a parallel architecture [9], to test various optimization functions and solve (3.8).

B) The theory of linear programming with multiple objectives can be invoked to tackle our optimization problem, where we have two kinds of cost (loss and delay) that we wish to minimize simultaneously.

C) One can introduce extra constraints in terms of the equilibrium state probabilities to the optimization problem in a direct way.

The LP formulation is easily obtained by again allowing nondeterministic but stationary and history-independent policies:

Minimize:

$$
\sum_{x} \sum_{a} \pi_{x a} \cdot w_{x a}
$$

Subject to:

$$
\begin{aligned}
& \sum_{x} \sum_{a} \pi_{x a} \cdot T_{z(x, a), y}=\sum_{a} \pi_{y a} \quad \forall y=1 \cdots \Xi \\
& \sum_{x} \sum_{a} \pi_{x a}=1 \\
& \pi_{x a} \geq 0 \quad \forall a \in K(x), \forall x=1 \cdots \Xi .
\end{aligned}
$$

$\pi_{r a}$ stands for $\operatorname{Pr}\{X(t)=x, A(t)=a\}$, whence $\pi_{x}=\Sigma_{a} \pi_{x t}$ and $\beta(x, a, h, t)=\pi_{x a} / \Sigma_{a} \pi_{x a}$. Accordingly, the constraints $(4.3 \mathrm{~b}-\mathrm{c})$ can be recognized as the familiar equilibrium probability equations. Since at least one of the constraints $(4.3 \mathrm{~b})$ is redundant, the number of effective constraints equals twice the number of states: $2 \cdot \Xi$. Also, since the Markov chain is irreducible, none of the equilibrium solutions can be zero: $\forall x: \Sigma_{a}$ $\pi_{x a}>0$. Since in an LP program, at most $\Xi$ (the number of basic variables) of the components of the solution vector can be positive, we are forced to conclude that, for exactly one $a, \forall x$ : $\pi_{x a}>0$ and $\pi_{x a}=0$ for all other $a$. In other words, the optimal policy indeed is a member of the class of deterministic policies. 
We now turn to our first problem: the determination of the objective function. Our goal is to minimize both delay variance of the $L D$ cells and the loss probability of $L L$ cells. So we have a multiple-objective optimization problem: the minimization of two cost functions, that both must be written in the form of (4.2). After motivating the equivalence of minimization of delay variance and minimization of expected delay (the latter can be put in the required form), we shall first find the two cost functions and then address the minimization tradeoff.

For the $L D$-cell delay variance, we used a pragmatic approach. Wanting to minimize delay variance, we strive after minimization of $\operatorname{Var}\left\{n_{L D}\right\}$ because generally, for almost any queueing system, a reduction of variance of the number of customers of a particular type is accompanied by a reduction of the variance of the delay. In Fig. 2 , a dot is placed in the $\left(E\left\{n_{L D}\right\}\right.$, $\operatorname{Var}\left\{n_{L D}\right\}^{1 / 2}$ ) plane for all (1024) deterministic LDOLL policies for given fixed parameters $Q, N, r, p$. The figure illustrates that minimization of $E\left\{n_{L D}\right\}$ leads to reduction of $\operatorname{Var}\left\{n_{L D}\right\}$. By the same token, minimization of mean $L D$-cell delay leads to reduction of $L D$-cell delay variance.

For the cost functions, we commence with expressing $L L$ cell loss probability. $L L$ cells are lost because of blocking, which happens due to lack of space for all arriving $L L$ cells in the LDOLL queue. We let $L_{L L}(t)$ denote the number of $L L$ cells that are lost during time slot $t$, and $\lim _{t \rightarrow \infty} E_{R}\left\{L_{L L}(t)\right\}$ $\stackrel{\Delta}{=}$

$$
\begin{aligned}
E_{R}\left\{L_{L L}\right\}= & N \cdot(1-r) \cdot p-\left\{(1-r \cdot p)^{N}\right. \\
& \left.-(1-p)^{N}\right\} \pi_{\varphi(0.0)}-\sum_{m=0}^{Q} \sum_{n=1}^{Q-m} \pi_{\varphi(m, n), a^{\prime}}
\end{aligned}
$$

Here $a^{\prime}$ is short for $(0,1)$, in words: "serve an $L L$ cell." The $R$ subscript signifies that the obtained expectation value is policy dependent, since the $\pi_{x a}$ depend on $R$. The first term in the expression is the offered $L L$ traffic, the second term is the expected number of cells served if the queue is empty because of the buffer bypass, and the third term is the expected number of $L L$ cells served per time slot. The $L L$-cell loss probability is

$$
\operatorname{Pr}_{R}\{L L \text {-cell lost }\}=\frac{E_{R}\left\{L_{L L}\right\}}{N \cdot(1-r) \cdot p} .
$$

This means that we can minimize the $L L$-cell loss probability by minimizing $E_{R}\left\{L_{L L}\right\}$, which is a linear combination of the state space probabilities, augmented with a constant, which is irrelevant for optimization. For matter of completeness, we note that $L D$ cells are lost for two reasons, blocking and replacement , so we have: $L_{L D}(t)=B_{L D}(t)+R_{L D}(t)$. In Appendix A, expressions for the probabilities of $L D$-cell loss and replacement can be found.

For the other cost function, we take, as was motivated earlier, expected $L D$-cell delay. In order to express the expected $L D$-cell delay as a function of the equilibrium state probabilities, we will have to use an approximation, the derivation of which may be found in Appendix B.

$$
\begin{aligned}
E_{R}\{L D \text {-cell delay }\} \\
\quad \approx \frac{E_{R}\left\{n_{L D}\right\}}{N \cdot r \cdot p \cdot\left(1-\operatorname{Pr}_{R}\{L D \text {-cell blocked }\}\right)}
\end{aligned}
$$

For calculation of $\operatorname{Pr}_{R}\{L D$ cell blocked $\}$, refer to Appendix A. For the reader, worried about a possibly unacceptable compilation of approximations, we recall the earlier statement that,

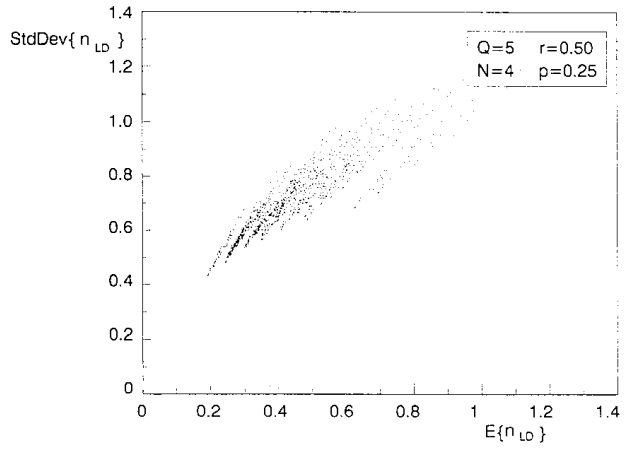

Fig. 2. Standard deviation versus expected number of $L D$ cell for 1024 different deterministic policies.

generally, a reduction of the number of customers is accompanied by a reduction of the delay variance.

Before we proceed, we recognize some general relations based on comparison of the deterministic policies $R$ with the FIFO queueing discipline. In a FIFO queue, the cell identity is not taken into consideration by the server. Mathematically, this means that the probability of serving an $L D$ cell if the system is in state $\left(n_{L D}, n_{L L}\right)$ is equal to $n_{L D} /\left(n_{L D}+n_{L L}\right)$. Also, neither type of cell has storage priority over the other, and cells cannot be replaced by other cells. A little reflection on the mechanics of the LDOLL queue gives us, for all deterministic LDOLL policies, $R$

$$
\begin{aligned}
E_{R}\left\{n_{L D}\right\}+E_{R}\left\{n_{L L}\right\} & =E_{\mathrm{FIFO}}\left\{n_{L D}+n_{L L}\right\} \\
E_{R}\left\{L_{L D}\right\}+E_{R}\left\{L_{L L}\right\} & =E_{\mathrm{FIFO}}\left\{L_{L L}+L_{L D}\right\} \\
E_{R}\left\{B_{L D}\right\} & =C
\end{aligned}
$$

with $C$ a constant that is independent of the $R$. This is so since the number of buffers that are free after $L L$ cells have been enqueued only depends on the total number of cells in the queue, which is independent of the policy. Therefore, we can minimize $L D$-cell delay simply by minimizing $E_{R}\left\{n_{L D}\right\}$, which can be expressed as a linear combination of equilibrium state probabilities as in (4.2).

In multiple objective optimization, of all feasible solutions [satisfying constraints $(4.3 \mathrm{~b}-\mathrm{d})$ ] only the so-called efficient solutions of the LP problem are considered. A solution is called an efficient solution in our problem if no feasible $\pi$ can be found that decreases $E_{R}\left\{n_{L D}\right\}$ without increasing $E_{R}\left\{L_{L L}\right\}$, or the other way around. Geoffrion [6] found that a feasible solution $\pi$ is efficient if, and only if, there exist positive weight coefficients $\lambda, \mu$ such that $\pi$ minimizes $\left[\lambda \cdot E_{R}\left\{n_{L D}\right\}+\mu\right.$. $E_{R}\left\{L_{L L}\right\}$ ], subject to $(4.3 \mathrm{~b})-(4.3 \mathrm{c})$.

The tradeoff between both objective functions achieved by this compound objective function can be visualized by considering the two-dimensional objective space with points $\left(E_{R}\left\{n_{L D}\right\}, E_{R}\left\{L_{L L}\right\}\right)$, as in Fig. 3. For a small LDOLL queue with arbitrary fixed $N, r$, and $p$, the solution was computed for every deterministic policy. Every dot marks the resultant $E_{R}\left\{n_{L D}\right\}$ and $E_{R}\left\{L_{L L}\right\}$. Obviously, the ratio of $\lambda$ and $\mu$ determines the slope of the linear contours of the objective function, so that modulation of this ratio allows us to reach different efficient solutions. The slope in the figure was obtained by setting $\lambda=\left(E_{\mathrm{FIFO}}\left\{n_{L D}\right\}\right)^{-1}, \mu=\left(E_{\mathrm{FIFO}}\left\{L_{L L}\right\}\right)^{-1}$. As a result, a small 


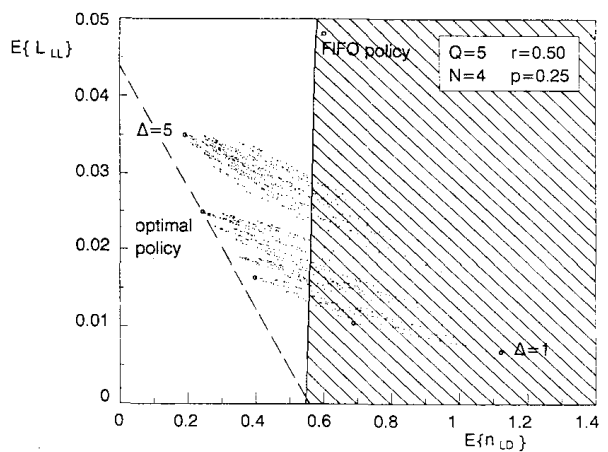

Fig. 3. Objective space with all 1024 deterministic policies. The encircled points correspond with LDOLL threshold policies. Policies that are fair (to be defined at the end of this section) lie outside the shaded area. The dashed line indicates a contour line where the weighted sum of both objectives is constant.

$E\{L D$-cell delay $\} / \operatorname{Pr}\{L L$-cell loss $\}$ under FIFO policy will be retained under LDOLL policy.

For increasing queue sizes, the size of the LP program grows quadratically. The number of variables is $Q^{2}+Q+1$ and the number of independent constraints is $(Q+1) \cdot(Q+2)$. The maximum queue size we can handle is restricted by the LP optimizer that is used. However, the number of policies can be reduced by taking into account only the efficient solutions. To that end, we need to know which policies can be dropped as effective solution candidates. We postulate that the efficient solutions can be easily identified.

Conjecture 1: The solution of (3.8) under policy $R(\Delta)$, where $R(\Delta)$ is the policy that gives service priority to $L D$ cells as long as $n_{L L}<\Delta$ and service priority to $L L$ cells whenever $n_{L L} \geq \Delta$, is an efficient solution to the LP problem (4.3) with objective functions $E_{R}\left\{n_{L D}\right\}$ and $E_{R}\left\{L_{L L}\right\}$.

We call $R(\Delta), \Delta=1 \cdots Q$, LDOLL threshold policies. Fig. 4 portrays this policy in the state space by means of arrows indicating the state transition effected by the server. Mathematically, the policy is defined by its action in state $x$ as follows:

$$
A(x)= \begin{cases}(0,0) & \text { if } n_{L D}=0 \text { and } n_{L L}=0 \\ (1,0) & \text { if } n_{L D}>1 \text { and } n_{L L}<\Delta \\ (0,1) & \text { if } n_{L D}=0 \text { and } n_{L L}>1 \\ (0,1) & \text { otherwise. }\end{cases}
$$

An increment of the threshold $\Delta$ of an LDOLL threshold policy yields a reduction of the expected $L D$-cell delay and an increase of the $L L$-cell loss probability.

Conjecture 2: The points $\left(E_{R(\Delta)}\left\{n_{L D}\right\}, E_{R(\Delta)}\left\{L_{L L}\right\}\right), \Delta=$ $1, \cdots, Q$ in the objective space are the vertices of the lower (south-west) convex hull of the mapping of the solution set onto the objective space.

Corollary: For the minimization of the compound objective function, only $Q$ policies will have to be considered. We can start with a threshold policy with, for example, $\Delta=Q / 2$ and calculate the corresponding $L D$-cell delay and $L L$-cell loss probability, and depending on whether we want to emphasize loss or delay reduction, accordingly adapt the value of $\Delta$.

In some cases, we can identify a lower bound on the value of $\Delta$, which means that we can cut down the number of optimal

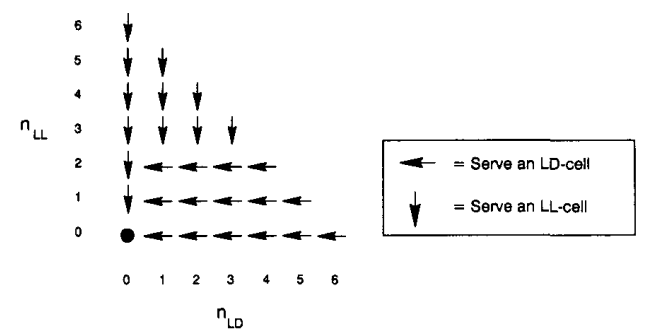

Fig. 4. Graphical representation of an LDOLL threshold policy with threshold $\Delta=3$ and queue size $Q=6$.

policies even further. Choosing a small $\Delta$ indeed yields a small $L L$-cell loss probability, but it also may increase the expected $L D$-cell delay to exceed the expected value of the $L L$-cell delay. In this way, fairness is lost; only low loss traffic profits from the LDOLL threshold policy. $L L$ cells have less delay, as well as a smaller loss probability than $L D$ cells. In Fig. 3, the part of the objective space where $E\{L D$-cell delay $\}>E\{L L$ cell delay $\}$ is shaded. The equation of the curve, where both expected delays match, follows from equating the $L L$ cell delay, which follows with Little's result:

$$
\begin{aligned}
& E_{R}\{L L \text {-cell delay }\} \\
& \quad=\frac{E_{R}\left\{n_{L L}\right\}}{N \cdot(1-r) \cdot p \cdot(1-\operatorname{Pr}\{L L \text {-cell lost }\})}
\end{aligned}
$$

to (4.6) and, using (4.7a) and (4.7c), giving

$$
\begin{aligned}
E_{R}\left\{L_{L L}\right\}= & (N \cdot p-C)-(N \cdot r \cdot p-C) \\
& \cdot \frac{E_{\mathrm{FIFO}}\left\{n_{L D}+n_{L L}\right\}}{E_{R}\left\{n_{L D}\right\}} .
\end{aligned}
$$

Note that, once we have found the coordinates of a policy on the objective space, all characteristic parameters of interest follow directly by means of equations $(4.7 \mathrm{a}-\mathrm{c})$. So if we wish to, we can easily enforce extra constraints, like an upper bound on $L L$-cell delay or on $L D$-loss probability.

\section{The Benefits of LDOLL Queueing and Mixed \\ TRAFFIC}

In this section, we will look at a sample LDOLL queue with ten cell buffers and four input channels $(Q=10, N=4)$. We will assess the advantages of the LDOLL queueing policy for mixed traffic, and the effect of mixing traffic for an LDOLL queue.

We will only study the cases where the intensity of incoming traffic does not exceed $100 \%$ (i.e., $N p \leq 1$ ). When employed in an ATM switching network, overload of an LDOLL queue should be avoided during normal operation by properly dimensioning of the network switches and transmission resources. However, with envisaged queue sizes of several hundreds of cell buffers, short periods of overload can be tolerated.

To begin with, we consider the situation where the incoming traffic streams contain $L D$ and $L L$ traffic in equal amounts. In order to select an appropriate service policy, we first take a look at the delay and loss characteristics of the LDOLL threshold policies in the objective space diagram (see Fig. 5). We take the case of maximal loading $(N p=1)$. From the figure, we conclude that the threshold policies with $\Delta$ ranging from 4 to 10 are fair; they do not favor $L L$ cells only. If $p$ is decreased, 


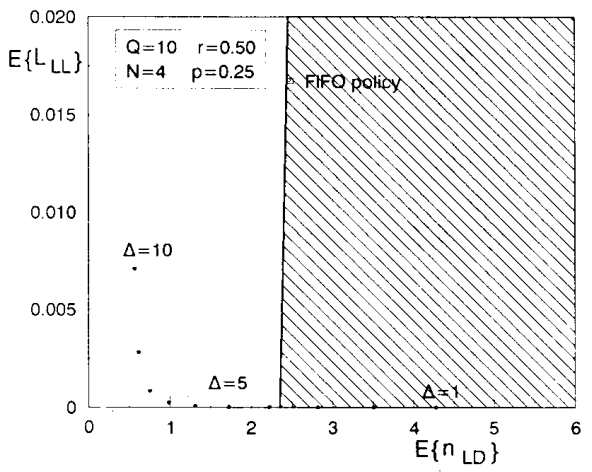

Fig. 5. Objective space with LDOLL threshold policies at $100 \%$ loading

the lower convex hull will shift towards the origin of the objective space, guaranteeing that the threshold policies with $\Delta \geq 4$ will indeed remain fair over the total considered range of $p=$ $0 \cdots 0.25$. In order to investigate how the choice of $\Delta$ affects the characteristics of the LDOLL queue, we shall study three cases. The extreme policies: a low threshold $\Delta=1$, a high threshold $\Delta=Q$, and an in-between policy with medium threshold $\Delta=5$.

In Fig. 6(a)-(c), the loss probabilities for both cell types (given by (4.5) and Appendix A) have been plotted as a function of input traffic intensity. Note that as $\Delta$ decreases, the $L L$-cell loss probability decreases over the whole range of considered $p$. The $L D$-cell loss probability seems unaffected by the change of policy, despite relation (4.7b). This effect is a consequence of the logarithmic scaling of the loss probabilities.

For the average cell delay, the tradeoff between both cell types becomes more clearly visible. Comparing $\Delta=5$ in Fig. 6(e) with $\Delta=10$ in Fig. 6(f) we see how a reduction of the $L D$ cell delay is paid with an increase in $L L$-cell delay. In Fig. 6(d), we note that for $N p$ greater than about 0.5 , the threshold policy with $\Delta=1$ is not fair. By making $p$ smaller, we "drag" the lower convex hull in the objective space over the boundary of (4.10) and fairness is restored.

Before studying the advantage of mixing traffic, we recognize that the mutual position of the loss curves and the delay curves does not differ essentially with varying $p$. Therefore, we will explore the relation between loss probability and mixing ratio $r$ and between average delay and $r$, for a fixed value of $N p$, say 0.7 . Both graphs can be found in Fig. 7. We observe that if $r$ $\rightarrow 0$ (for $r=0 \operatorname{Pr}\{L D$-cell loss $\}$ has no meaning) the $L L$ cell loss probability approaches the cell loss probabilities for a FIFO queue. For unmixed traffic streams, the LDOLL queue behaves like a FIFO queue, naturally. For the same reason, the $L D$-cell loss probability also approaches the FIFO cell loss probability as $r \rightarrow 1$. The $L L$-cell loss probability drops to zero because of the replacement mechanism. To the $L L$ cells-that incidentally arrive if $r \approx 1$-the LDOLL queue is virtually empty, since they have storage priority over $L D$ cells.

For the $L D$ cell and $L L$ cell delay, the FIFO properties are also matched as $r$ approaches 1 and 0 , respectively. The corresponding limit values of $L L$ cell and $L D$ cell delay in these cases are both nonzero.

\section{CONCLUSIONS}

It is advantageous, as is shown in the preceding sections, to consider two different classes of ATM cells. Each cell class has its own objective: low delay and low delay variance for one class, and low loss probability for the other. The CLP (cell loss priority) bit, as defined by CCITT SG XVIII, could conceivably be used to identify both classes. The ATM switching network distinguishes and distinctively handles cells of either type. We considered an ATM network being built of basic switching elements that employ a very general store and forward switching policy. This element can operate according to a very large class of policies. We successively reduced the class of feasible policies that render optimization of the objectives, to the class of so-called LDOLL threshold policies, containing exactly $Q$ policies, $Q$ being the number of cell buffers in the switching element. We introduced the term fairness, which refers to both cell types profiting from the LDOLL policy and not just low loss traffic.

The performance of a cell type improves the more this type is in minority. This effect is most articulate for low loss $(L L)$ cells. In a typical case, the loss probability goes from $10^{-6}$ with $80 \% L L$ cells to $10^{-12}$ with $20 \% L L$ cells. On the same range, the delay of $L D$ cells halves and the delay variance shows a corresponding reduction. This clearly demonstrates the benefit of LDOLL threshold policies in the context of mixed traffic. In the objective space, any deterministic LDOLL policy compares favorably with the FIFO policy. Obviously, when the mixing ratio $r \rightarrow 0$ or $r \rightarrow 1$, the performance of the majority cell type converges to FIFO performance.

Preliminary results indicate that, with sufficient buffer space, an LDOLL threshold policy with fixed threshold is robust. By robustness, we mean the insensitivity of the optimal policy to sudden periods of overload, or periods of imbalance, where the traffic is momentarily unmixed. These sudden variations are due to the burstiness of the input traffic, which is modeled on the ACTION level (Section I). The results assessed in Section V already showed that a particular LDOLL threshold policy can be optimal for a large range of $p$. This aspect of robustness is currently being investigated. Also for further research in the cooperation of traffic control at different levels: from LDOLL policies at the TRANSMISSION (or CELL) level to call acceptance control at the CONNECTION level.

\section{APPENDIX A}

EXPRESSIONS FOR $L D$ CELl LOSS AND RePlacement Probabilities

The expression for the mean number of $L D$ cells lost is very similar to (4.4):

$$
\begin{aligned}
E_{R}\left\{L_{L D}\right\}= & N \cdot r \cdot p-\left\{1-(1-r \cdot p)^{N}\right\} \pi_{\varphi(0,0)} \\
& -\sum_{m=1}^{Q} \sum_{n=0}^{Q-m} \pi_{\varphi(m, n), a^{\mu}} .
\end{aligned}
$$

The second term represents the expected number of $L D$ cells served by means of a buffer bypass. The "serve an $L D$ cell" action is $a^{\prime \prime}=(1,0)$. The average number of $L D$ cells replaced per time slot for deterministic policies can be calculated with:

$$
\begin{aligned}
E_{R}\left\{R_{L D}\right\}= & \sum_{m=0}^{Q} \sum_{n=1}^{Q-m} \sum_{j=0}^{N} \\
& \cdot \operatorname{Max}\{0, \operatorname{Min}\{m+n-1+j-Q, \\
& \left.\left.m-\beta\left(\varphi(m, n), a^{\prime \prime}\right)\right\}\right\} \\
& \cdot \operatorname{Pr}\left\{A_{L L}=j\right\} \cdot \pi_{\varphi(m, n) .}
\end{aligned}
$$




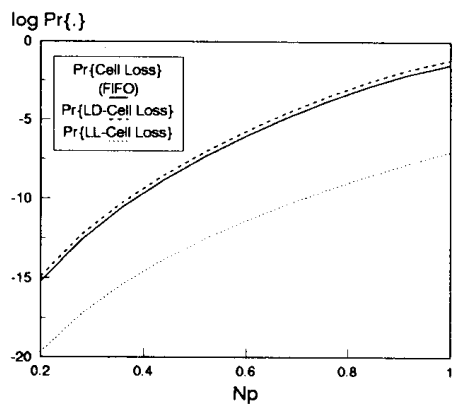

(a)

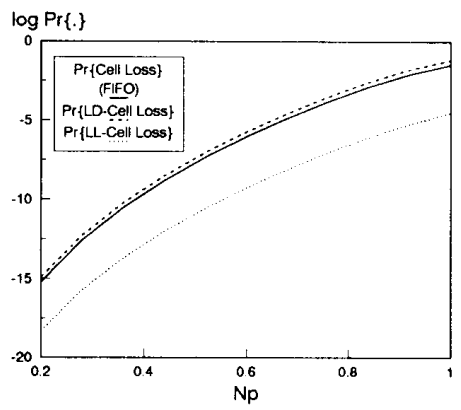

(b)

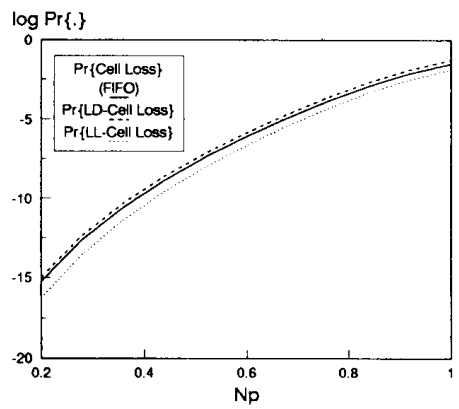

(c)

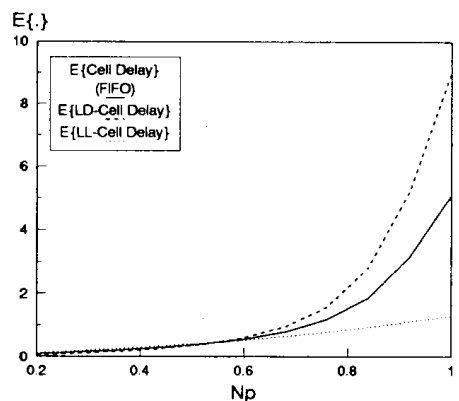

(d)

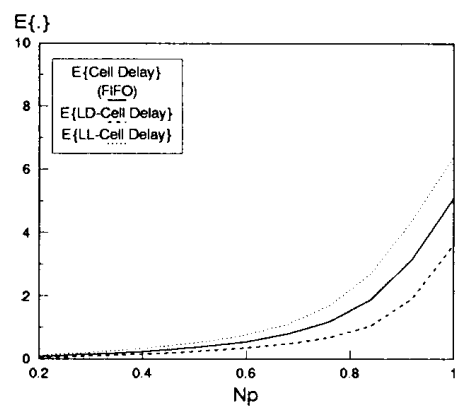

(e)

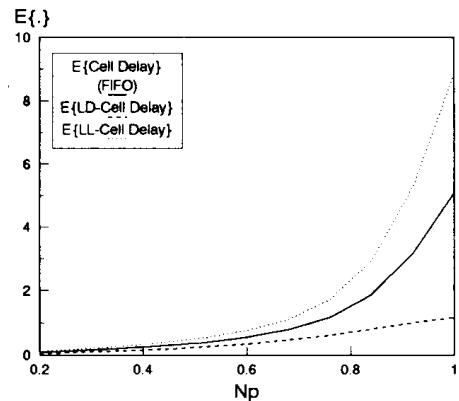

(f)

Fig. 6. Loss and delay properties as function of loading for various thresholds. (a) LDOLL loss probabilities for low threshold $\Delta=1 Q=10 N=4$. (b) LDOLL loss probabilities for medium threshold $\Delta=5 Q=10 N=4$. (c) LDOLL loss probabilities for high threshold $\Delta=10 Q=10 N=4$. (d) LDOLL average delays for low theshold $\Delta=1 Q=10-N=4$. (f) LDOLL average delays for high threshold $\Delta=10 Q=10 \mathrm{~N}=4$.

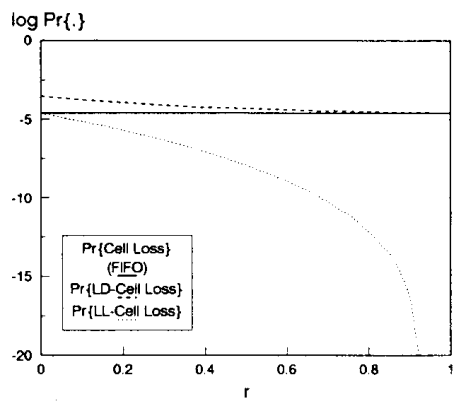

(a)

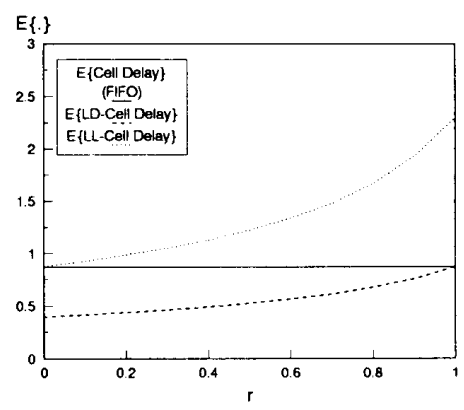

(b)

Fig. 7. Loss and delay properties for $N p=0.7$ as function of the mixing rato. (a) LDOLL loss prob. for varying mixing ratio $\Delta 5 Q=10 \mathrm{~N}=4$. (b) LDOLL average delays for varying mixing ratio $\Delta 5 Q=10 \mathrm{~N}=4$. 
$\operatorname{Pr}\left\{A_{L L}=j\right\}$ follows from (3.1) and has a binomial distribution. $E_{R}\left\{B_{L D}\right\}(\triangleq C$ ) is found by subtracting (A.2) from (A.1). The $L D$ cell replacement, blocking, and loss probabilities are obtained by dividing the corresponding expectation values by $N \cdot r \cdot p$.

\section{APPENDIX B}

Approximating Expression for Expected $L D$ Cell Delay

Here we want to find an expression for the delay of $L D$ cells. The delay is identical to the time spent in the LDOLL queue. Input buffering and transmission delay are not accounted for as delay. Cells that are replaced do not have a delay.

Define $\gamma(t)$ as the accumulated customer time of all $L D$ cells in the LDOLL queue, regardless of whether they are (to be) served or (to be) replaced. Likewise, define $\vartheta(t)$ as the accumulated customer time of all $L D$ cells that are (to be) replaced.

$$
\begin{aligned}
& \gamma(t) \triangleq \int_{0}^{t}[\alpha(\tau)-\delta(\tau)] d \tau \\
& \vartheta(t) \triangleq \sum_{i=0}^{\rho(t)} T_{i}
\end{aligned}
$$

where $T_{i}$ denotes the delay time of the $i$ th $L D$ cell that is (to be) replaced. The cumulative number of arrivals $\alpha$, departures $\delta$, replacements $\rho$, and services $\sigma$ are defined as

$$
\begin{aligned}
\alpha(t) & \triangleq \sum_{\tau=0}\left[A_{L D}(\tau)-B_{L D}(\tau)\right] \\
\rho(t) & \triangleq \sum_{\tau=0}^{t} R_{L D}(\tau) \\
\sigma(t) & \triangleq \sum_{\tau=0}^{t} S_{L D}(\tau) \\
\delta(t) & \triangleq \sigma(t)+\rho(t) .
\end{aligned}
$$

We can express the average $L D$-cell delay up to time slot $t$ as follows:

$$
E\{L D \text {-cell delay }\}(t)=\frac{\gamma(t)-\vartheta(t)}{\sigma(t)} .
$$

We see that, in order to minimize the average $L D$ cell delay, that we must make $\vartheta$ as large as possible. Fig. 8 shows that we must make the shaded areas as large as possible, which is achieved by replacing the oldest $L D$ cell(s) first. We rewrite (B.3) to obtain

$$
E\{L D \text {-cell delay }\}(t) \cdot \frac{\sigma(t)}{t}=\frac{\gamma(t)}{t}-\frac{\vartheta(t)}{\rho(t)} \cdot \frac{\rho(t)}{t} .
$$

Taking the limit as $t \rightarrow \infty$ on both sides of this equation yields

$$
\begin{gathered}
E_{R}\{L D \text {-cell delay }\} \cdot\left(N \cdot r \cdot p-E\left\{B_{L D}\right\}-E\left\{R_{L D}\right\}\right) \\
=E\left\{n_{L D}\right\}-\bar{T} \cdot E\left\{R_{L D}\right\} .
\end{gathered}
$$

$\bar{T}$ denotes the average delay that is experienced by $L D$ cells which will eventually be replaced. If we assume that replacement of more than one $L D$ cell per time slot is a rare event, the head-of-line cell (the oldest cell in the line of $L D$ cells in the LDOLL queue ) almost always gets replaced. Therefore, a head-

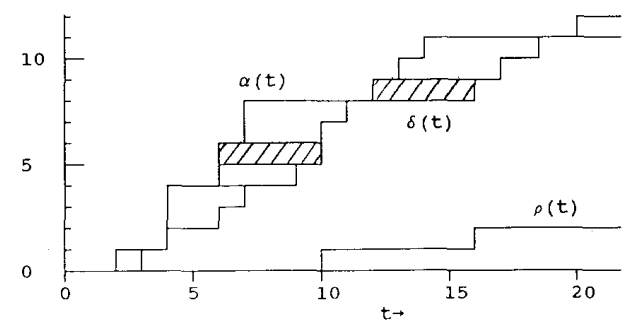

Fig. 8. Sample realization of arrival, service, and loss process.

of-line $L D$ cell stays in the queue until it is served or replaced. In both cases, the time spent in the LDOLL queue will be equal, on the average. For that reason, we set $\bar{T}$ equal to $E\{L D$ cell delay $\}$. By doing so, we get (4.6) for the expected $L D$ cell delay.

\section{REFERENCES}

[1] CCITT SG XVIII, Draft Recommendation I.361, "ATM layer specification for B-ISDN," Rep. XVIII-R 23-E, Geneva, Jan. 1990.

[2] CCITT, Recommendation I.121, "Broadband aspects of ISDN," Blue Book, Fascicle III.7, Melbourne, 1988.

[3] J. P. Coudreuse, "ATM: A contribution to the debate on broadband ISDN," Int. J. Digit. Analog Cabled Syst., vol. 1, pp. 213$221,1988$.

[4] C. Derman, "Finite state Markovian decision processes," Mathematics in Science and Engineering. New York: Academic, 1970 , vol. 67.

[5] J. Filipiak, "Structured systems analysis methodology for design of an ATM network architecture," IEEE J. Select. Areas Commun., vol. 7, pp. 1263-1273, Oct. 1989.

[6] A. M. Geoffrion, "Proper efficiency and theory of vector maximization," J. Math. Anal. Appl., vol. 22, pp. 618-630, 1968.

[7] J. Y. Hui, "Resource allocation for broadband networks," IEEE J. Select. Areas Commun., vol. 6, pp. 1598-1608, Dec. 1988.

[8] M. J. Karol, M. G. Hluchyj, and S. P. Morgan, "Input versus output queueing on a space-division packet switch," IEEE Trans. Commun, vol. COM-35, pp. 1347-1356, Dec. 1987.

[9] J. Luo, G. L. Reijns, and F. Bruggeman, "A flexible transputer network for numerical applications," The EuroMicro Journal: Microprocessing and Microprogramming, vol. 27, pp. 405-412, 1989.

[10] A. S. Manne, "Linear programming and sequential decisions," Mgt. Sci., vol. 6, pp. 259-267, 1960.

[11] H. Ohnishi, T. Okada, and K. H. Noguchi, "Flow control schemes and delay/loss tradeoff in ATM networks," IEEE J. Select. Areas Commun., vol. 6, pp. 1609-1616, Dec. 1988.

[12] F. C. Schoute and G. A. Awater, "Performance improvement of fast packet switching by LDOLL queueing," to be presented at Proc. ITC 13, Copenhagen, Denmark, June 1991.

[13] F. C. Schoute, "Simple decision rules for acceptance of mixed traffic streams," in Proc. ITC 12, Torino, Italy, June 1988, pp. 4.2A.5.1-7.

[14] F. C. Schoute, "Mixed traffic patterns and traffic capacity in ISDN," in Proceedings IFP TC 6/ICC Joint Conference on ISDN in Europe, J. Arnbak, Ed. North Holland: Elsevier, 1989, pp. 97-105.

[15] J. S. Turner, "Design of a broadcast packet switching network," IEEE Trans. Commun., vol. 36, pp. 734-743, June 1988.

[16] W. Verbiest and L. Pinnoo, "A variable bit rate codec for asynchronous transfer mode networks," IEEE J. Select. Areas Commun., vol. 7, pp. 761-770, June 1989.

[17] W. Verbiest, L. Pinnoo, and B. Voeten, "The impact of the ATM concept on video coding," IEEE J. Select. Areas Commun., vol. 6, pp. 1623-1632, Dec. 1988. 


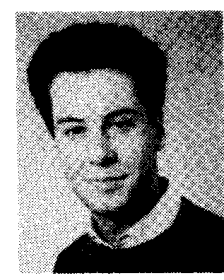

Geert A. Awater was born in 1965. He graduated from Twente University of Technology, Netherlands, with a degree in electrical engineering in 1989

In the same year, he joined the Telecommunications and Traffic Control Systems Group, Department of Electrical Engineering, Delft University of Technology, where he is working towards his Ph.D. degree. He is currently engaged in research on the teletraffic theory of broadband switching networks and on broadband videotelephony.

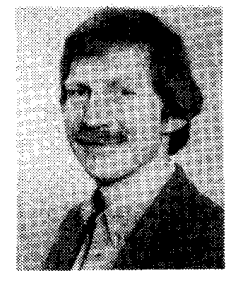

Frits C. Schoute received the Masters degree in applied mathematics from the University of Groningen, Netherlands, in 1974. In 1977 he obtained the $\mathrm{Ph} . \mathrm{D}$. degree in engineering from Harvard University, Cambridge, MA, with thesis on decentralized control in computer communication.

Since then, he has been with Philips Telecommunication in Hilversum, with the exception of the academic year ' 84 /' 85 , when he was Visiting Lecturer in the EECS department of the University of California at Berkeley. In 1989 he was appointed part-time professor in the Telecommunications and Traffic Control Systems Group (the Department of Electrical Engineering) of the Delft University of Technology. He teaches and researches in the area of performance analysis of telecommunication systems. 